\begin{tabular}{|c|c|c|c|c|c|c|}
\hline Impact Factor: & $\begin{array}{l}\text { ISRA (India) } \\
\text { ISI (Dubai, UAF } \\
\text { GIF (Australia) } \\
\text { JIF }\end{array}$ & $\begin{array}{l}=1.344 \\
=0.829 \\
=0.564 \\
=1.500\end{array}$ & $\begin{array}{l}\text { SIS (USA) } \\
\text { PИНЦ (Russia) } \\
\text { ESJI (KZ) } \\
\text { SJIF (Morocco) }\end{array}$ & $\begin{array}{l}=0.912 \\
=0.234 \\
=1.042 \\
=2.031\end{array}$ & $\begin{array}{l}\text { ICV (Poland) } \\
\text { PIF (India) } \\
\text { IBI (India) }\end{array}$ & $\begin{array}{l}=6.630 \\
=1.940 \\
=4.260\end{array}$ \\
\hline
\end{tabular}

\section{International Scientific Journal Theoretical \& Applied Science}

\author{
p-ISSN: 2308-4944 (print) e-ISSN: 2409-0085 (online) \\ Year: $2016 \quad$ Issue: 6 Volume: 38 \\ Published: $30.06 .2016 \quad \underline{\text { http://T-Science.org }}$
}

SECTION 2. Applied mathematics. Mathematical modeling.
Alexandr Shevtsov candidate of technical sciences, member of PILA (USA), member of European Academy of Natural History (UK), corresponding member of the Kazakhstan National Academy of Natural Sciences, President of International Academy TAS, Department of «Mathematics», Deputy Director on Science of faculty of information technologies, automation and telecommunications, Taraz state University named after M.Kh. Dulati, Kazakhstan Shev_AlexXXXX@mail.ru

\title{
SOME METHODS OF DIFFERENTIATION OF LAYERS IN DELPHI
}

\author{
Abstract: This article discusses some methods of isolation layers in Delphi, in relation to the geodetic satellite \\ maps. \\ Key words: layer, maps, delphi. \\ Language: English \\ Citation: Shevtsov AN (2016) SOME METHODS OF DIFFERENTIATION OF LAYERS IN DELPHI. ISJ \\ Theoretical \& Applied Science, 06 (38): 155-165. \\ Soi: http://s-o-i.org/1.1/TAS-06-38-32 Doi: crossef http://dx.doi.org/10.15863/TAS.2016.06.38.32

\section{Introduction} \\ In a separate geodetic tasks there is the problem \\ of re-projection and allocation of separate cultures \\ (layers) of a satellite map which shows several dozen \\ of crops. [1-7] \\ - Load arbitrary map image (Fig.1) \\ - Re-projection and allocation of the common \\ image block of $100 \times 100 \mathrm{~km}$ \\ - Split into separate layers, and the allocation of \\ each layer \\ - Save the obtained results
}

\section{Model}

Let's will develop a program in Delphi which will work with large amounts of graphical information and will perform the following tasks:

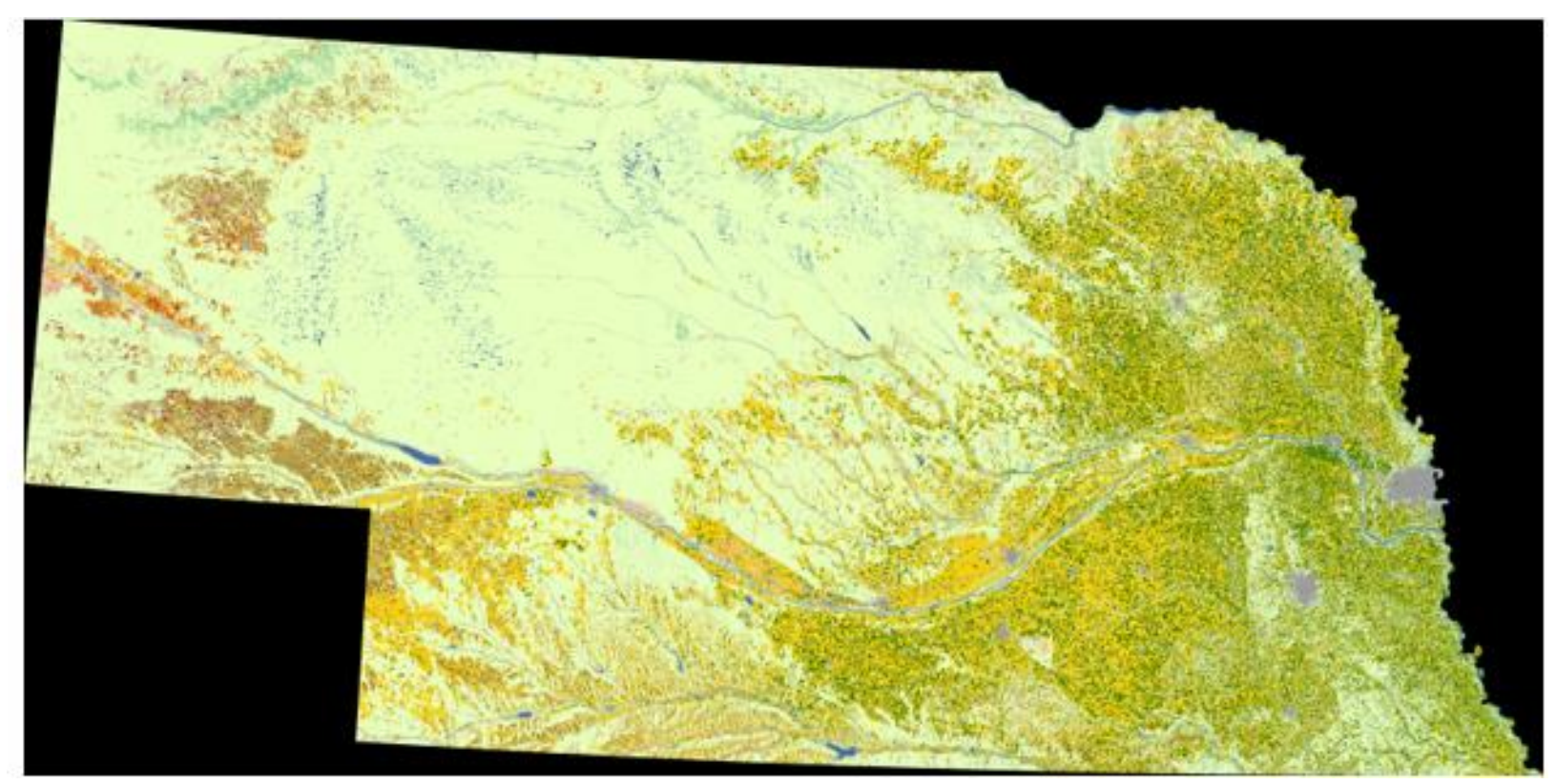

Figure 1 - Original image with all layers. 


\begin{tabular}{|c|c|c|c|c|c|c|}
\hline Impact Factor: & $\begin{array}{l}\text { ISRA (India) } \\
\text { ISI (Dubai, UAF } \\
\text { GIF (Australia) } \\
\text { JIF }\end{array}$ & $\begin{array}{l}=1.344 \\
=0.829 \\
=0.564 \\
=1.500\end{array}$ & $\begin{array}{l}\text { SIS (USA) } \\
\text { PИНЦ (Russia) } \\
\text { ESJI (KZ) } \\
\text { SJIF (Morocco) }\end{array}$ & $\begin{array}{l}=0.912 \\
=0.234 \\
=1.042 \\
=\mathbf{2 . 0 3 1}\end{array}$ & $\begin{array}{l}\text { ICV (Poland) } \\
\text { PIF (India) } \\
\text { IBI (India) }\end{array}$ & $\begin{array}{l}=6.630 \\
=1.940 \\
=4.260\end{array}$ \\
\hline
\end{tabular}

\section{Materials and methods}

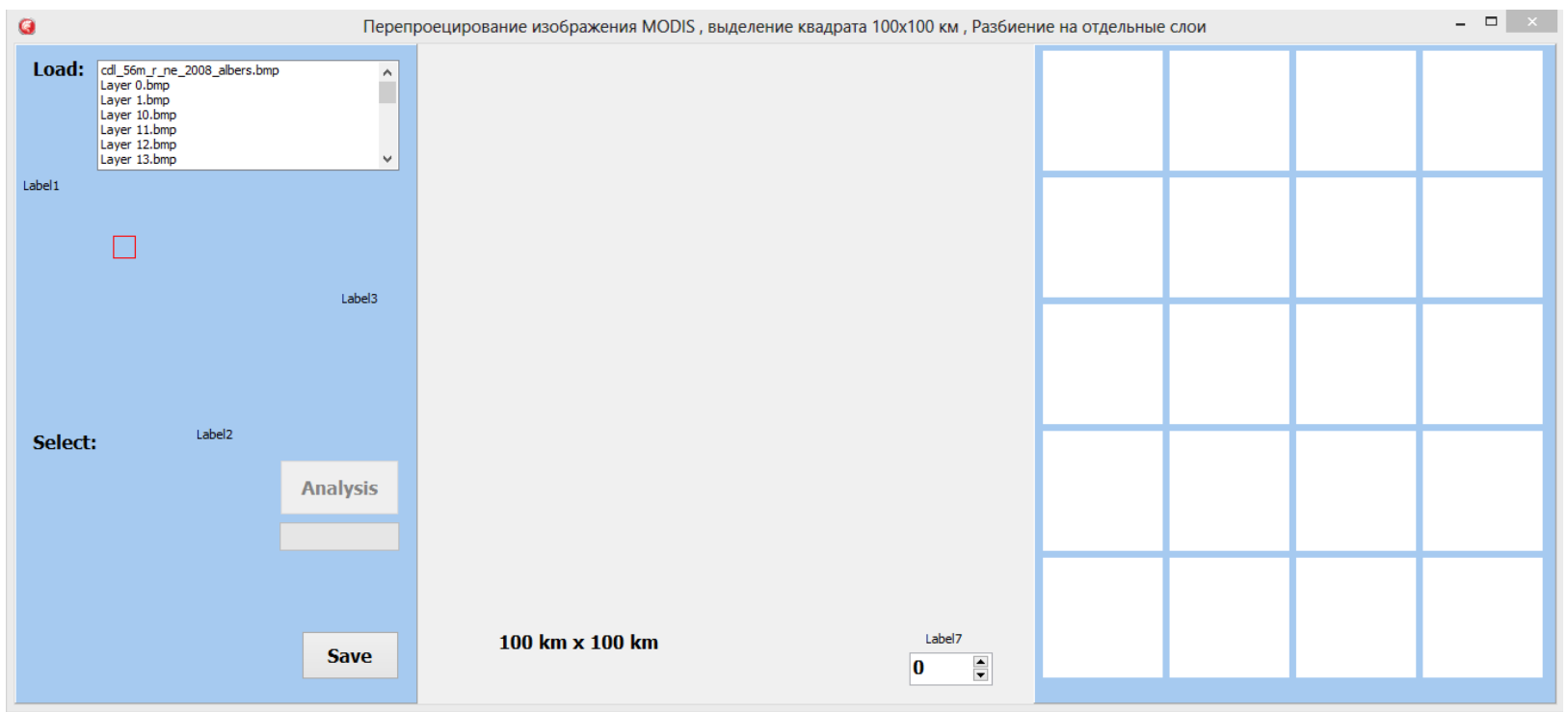

Figure 2 - The form of the program.

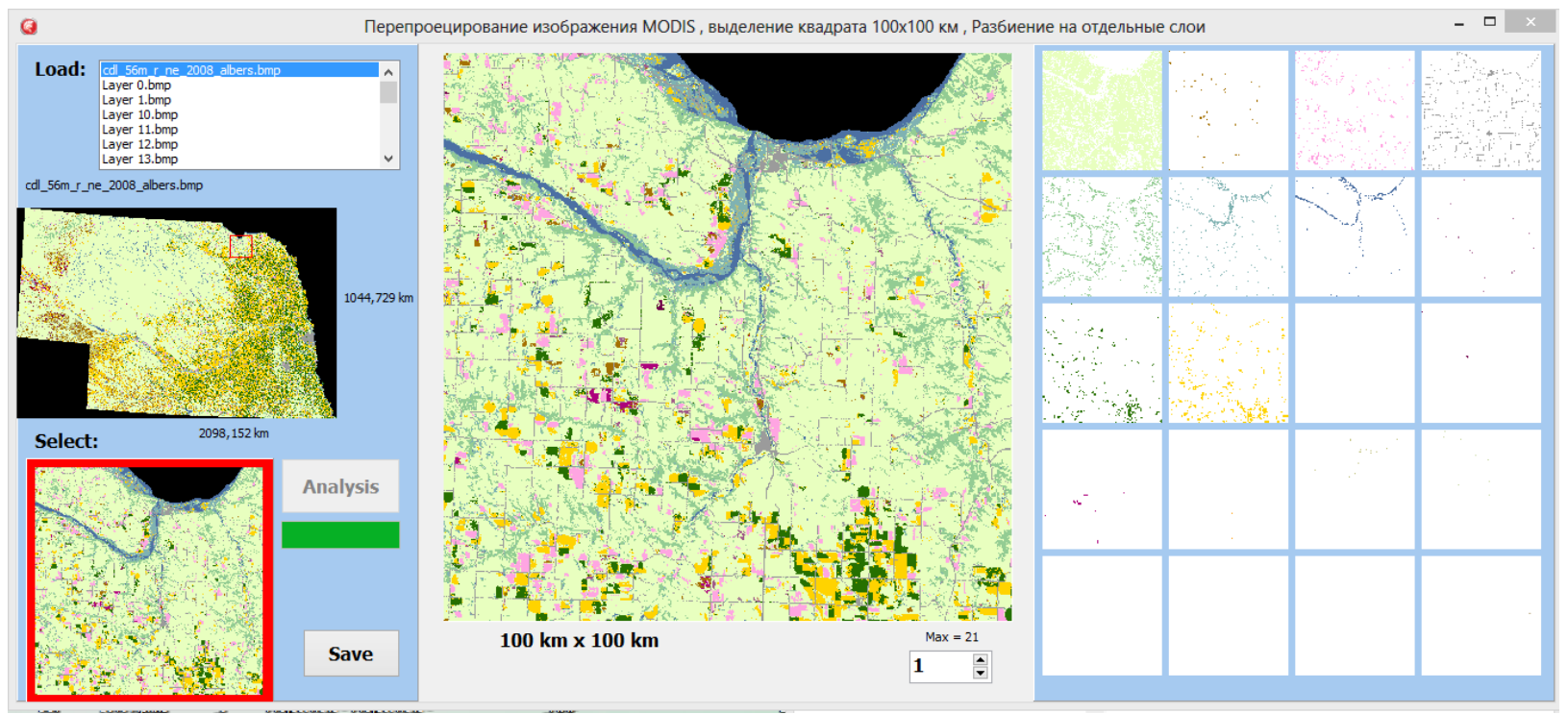

Figure 3 - The selection of layers.

In the program window (Fig. 3), it is possible to select a region on the map size of $100 \times 100 \mathrm{~km}$. Then, the obtained image becomes a separate and stored (Fig.4) Split it into layers occurs through the analysis and selection of certain shades of color. Each individual layer is stored in an array and is displayed on the right panel of the window. 


\begin{tabular}{l|lr|ll|ll} 
& ISRA (India) & $=\mathbf{1 . 3 4 4}$ & SIS (USA) & $=\mathbf{0 . 9 1 2}$ & ICV (Poland) & $=\mathbf{6 . 6 3 0}$ \\
Impact Factor: & ISI (Dubai, UAE) $=\mathbf{0 . 8 2 9}$ & PUHЦ (Russia) $=\mathbf{0 . 2 3 4}$ & PIF (India) & $=\mathbf{1 . 9 4 0}$ \\
& GIF (Australia) & $=\mathbf{0 . 5 6 4}$ & ESJI (KZ) & $=\mathbf{1 . 0 4 2}$ & IBI (India) & $=\mathbf{4 . 2 6 0}$ \\
& JIF & $\mathbf{1 . 5 0 0}$ & SJIF (Morocco) & $\mathbf{2 . 0 3 1}$ & & \\
\hline
\end{tabular}

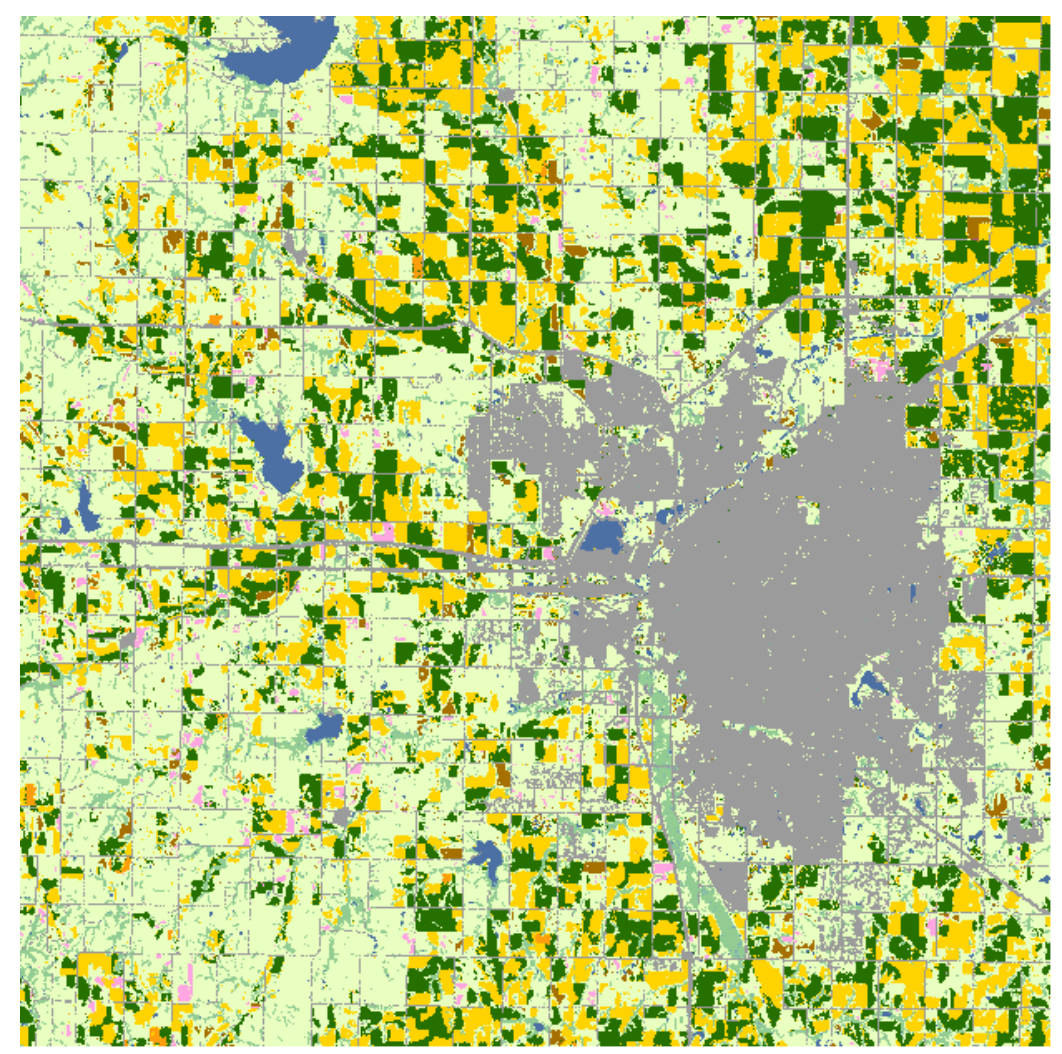

Figure 4 - The resulting image is $100 \times 100 \mathrm{~km}$.
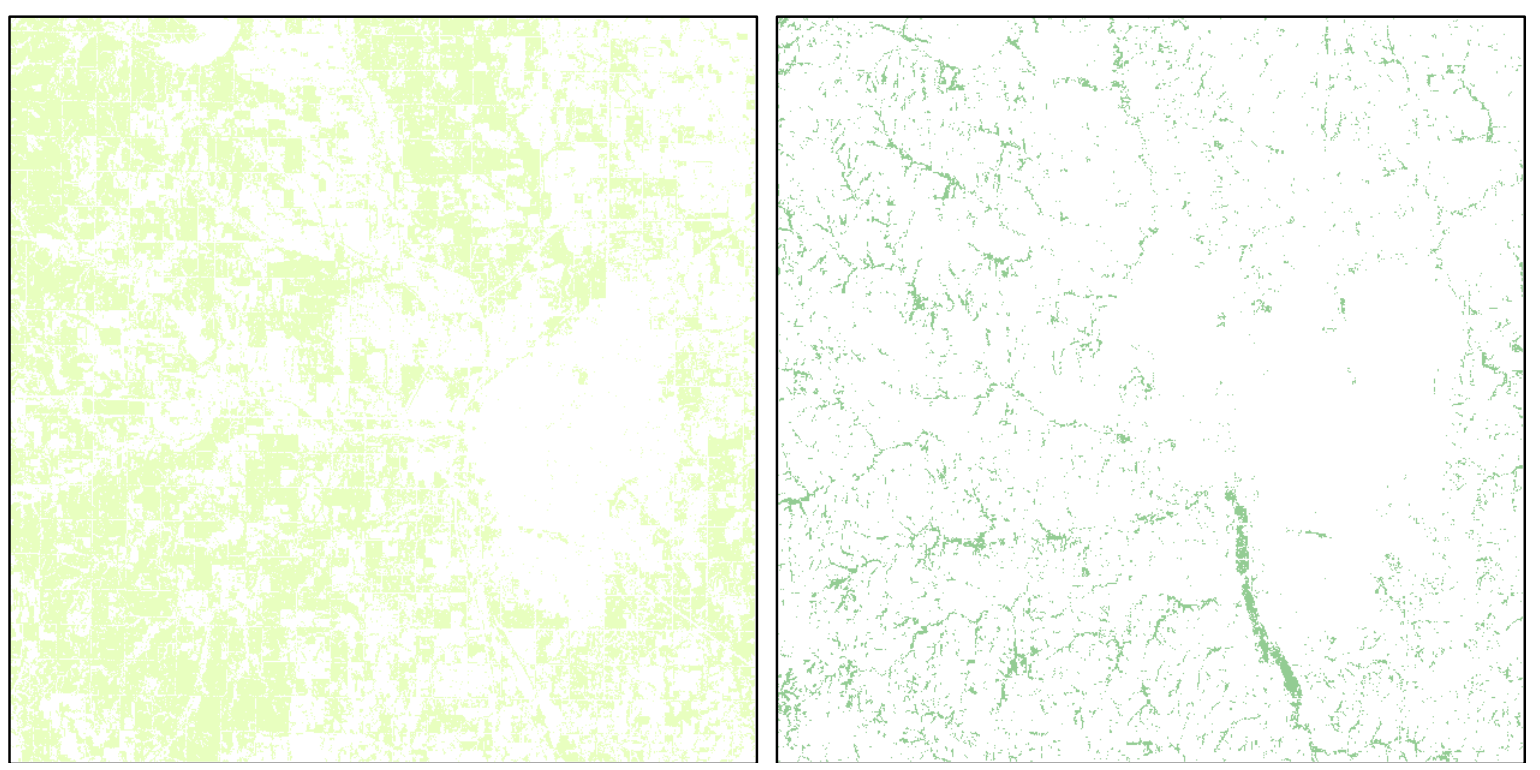

Figure 5 - The resulting layers 1-2.

The resulting layers characterize the crops grown in these areas. 


\begin{tabular}{|c|c|c|c|c|c|c|}
\hline Impact Factor: & $\begin{array}{l}\text { ISRA (India) } \\
\text { ISI (Dubai, UAE } \\
\text { GIF (Australia) } \\
\text { JIF }\end{array}$ & $\begin{array}{l}=1.344 \\
=0.829 \\
=0.564 \\
=1.500\end{array}$ & $\begin{array}{l}\text { SIS (USA) } \\
\text { PИНЦ (Russia) } \\
\text { ESJI (KZ) } \\
\text { SJIF (Morocco) }\end{array}$ & $\begin{array}{l}=0.912 \\
=0.234 \\
=1.042 \\
=\mathbf{2 . 0 3 1}\end{array}$ & $\begin{array}{l}\text { ICV (Poland) } \\
\text { PIF (India) } \\
\text { IBI (India) }\end{array}$ & $\begin{array}{l}=6.630 \\
=1.940 \\
=4.260\end{array}$ \\
\hline
\end{tabular}
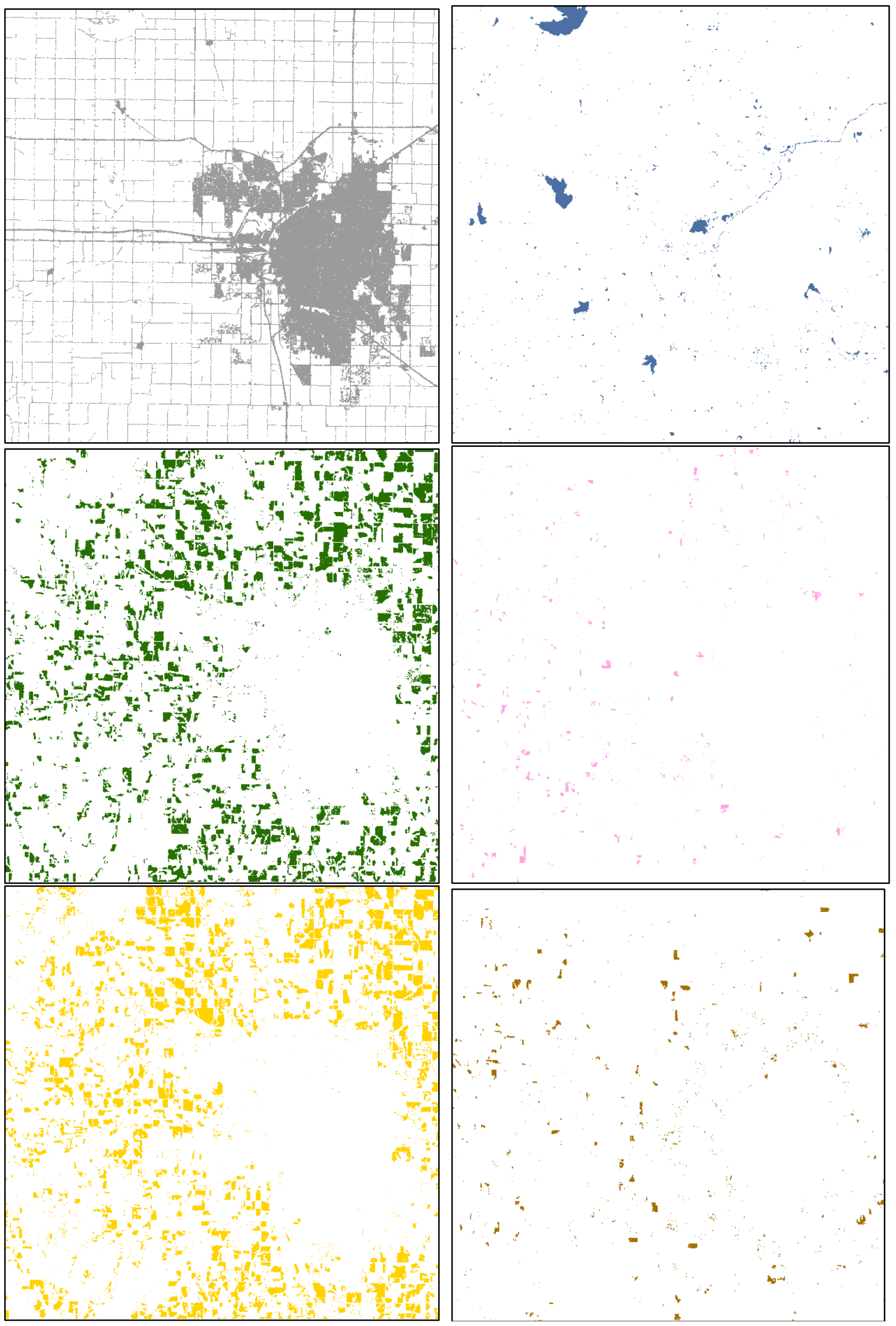

Figure 6 - The resulting layers 3-8.

ISPC Theory and Practice, 


\begin{tabular}{|c|c|c|c|c|c|c|}
\hline Impact Factor: & $\begin{array}{l}\text { ISRA (India) } \\
\text { ISI (Dubai, UAE } \\
\text { GIF (Australia) } \\
\text { JIF }\end{array}$ & $\begin{array}{l}=1.344 \\
=0.829 \\
=0.564 \\
=1.500\end{array}$ & $\begin{array}{l}\text { SIS (USA) } \\
\text { PИНЦ (Russia) } \\
\text { ESJI (KZ) } \\
\text { SJIF (Morocco) }\end{array}$ & $\begin{array}{l}=0.912 \\
=0.234 \\
=1.042 \\
=\mathbf{2 . 0 3 1}\end{array}$ & $\begin{array}{l}\text { ICV (Poland) } \\
\text { PIF (India) } \\
\text { IBI (India) }\end{array}$ & $\begin{array}{l}=6.630 \\
=1.940 \\
=4.260\end{array}$ \\
\hline
\end{tabular}
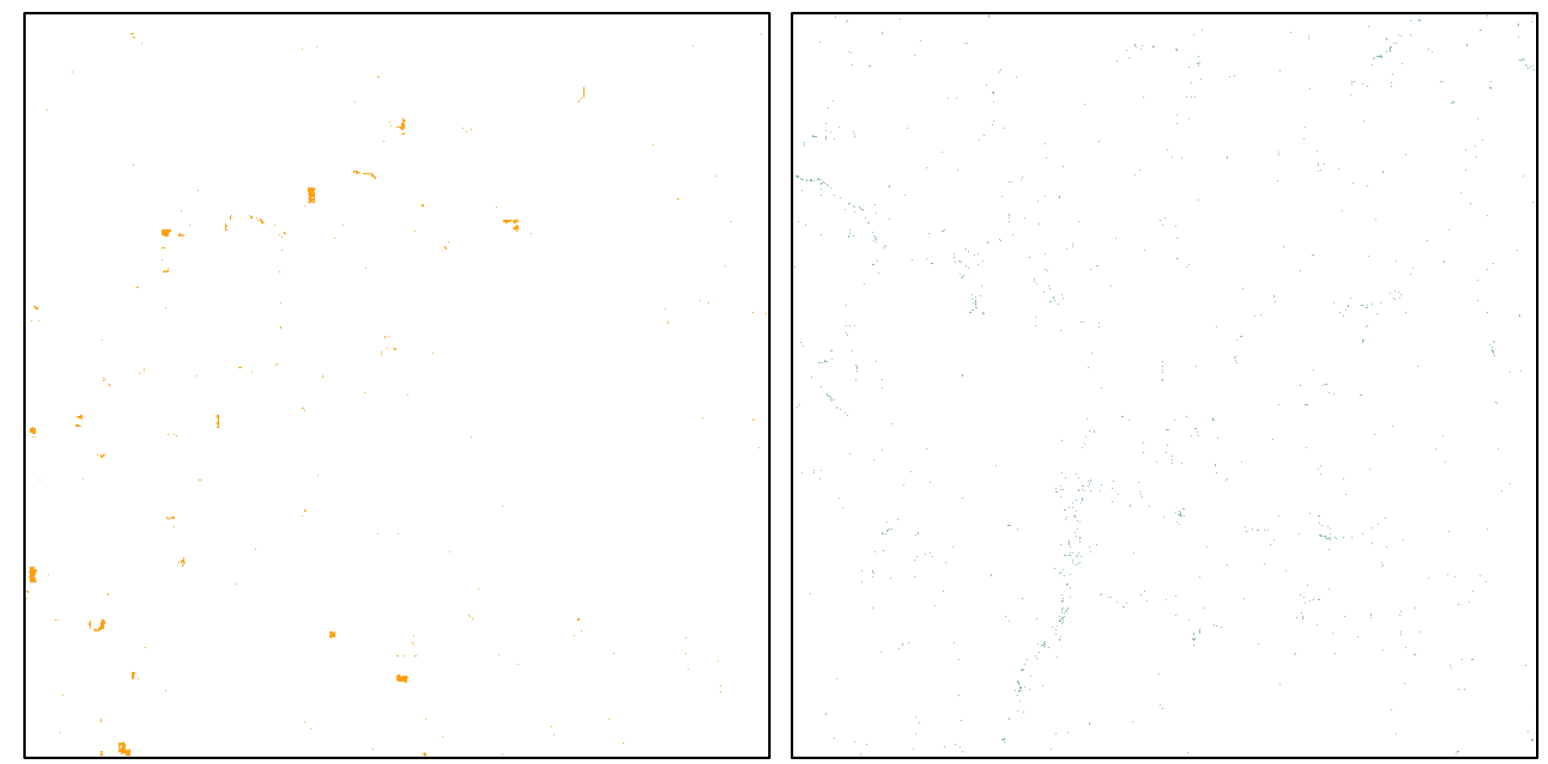

Figure 7 - The resulting layers 9-10.

\section{Results}

The developed program allows to quickly and easily select from maps of the area, with a plot size of $100 \times 100 \mathrm{~km}$ and divide it into separate layers. The obtained layers can be used to create temporary maps of cultivation of agricultural crops.

\section{References:}

1. (2016) Peredovye tekhnologii v obrabotke DDZ Available: $\quad$ http://www.estimap.ru/product/erdas (Accessed: 20.06.2016).

2. (2016) U.S. Geological Survey. Available: https://www.usgs.gov/ (Accessed: 20.06.2016).

3. (2016) Ortogonalizatsiya i dekorrelyatsiya vkhodnykh vektorov. Available: http://ww.helpiks.org/6-10663.html (Accessed: 20.06.2016).

4. (2016) Metod glavnykh komponent. Available: https://ru.wikipedia.org/wiki/Metod glavnykh komponent (Accessed: 20.06.2016).
5. (2016) Reproject using ERDAS Imagine. Available: http://mondegeospatial.com/reproject-using-erdas-imagine/ (Accessed: 20.06.2016).

6. (2016) Funktsiya Pereproetsirovat' (Reproject) Available: http://gis-lab.info/qa/reproject.html (Accessed: 20.06.2016).

7. (2016) Geographic Information Systems Stack Exchange Available: http://gis.stackexchange.com/ (Accessed: 20.06.2016).

The program in Delphi.

unit Unit1;

interface

uses

Winapi.Windows, Winapi.Messages, System.SysUtils, System.Variants, System.Classes, Vcl.Graphics, Vcl.Controls, Vcl.Forms, Vcl.Dialogs, Vcl.StdCtrls, Vcl.FileCtrl, Vcl.ExtCtrls, 


\begin{tabular}{|c|c|c|c|c|c|c|}
\hline Impact Factor: & $\begin{array}{l}\text { ISRA (India) } \\
\text { ISI (Dubai, UAF } \\
\text { GIF (Australia) } \\
\text { JIF }\end{array}$ & $\begin{array}{l}=1.344 \\
=0.829 \\
=0.564 \\
=1.500\end{array}$ & $\begin{array}{l}\text { SIS (USA) } \\
\text { PИНЦ (Russia) } \\
\text { ESJI (KZ) } \\
\text { SJIF (Morocco) }\end{array}$ & $\begin{array}{l}=0.912 \\
=0.234 \\
=1.042 \\
=2.031\end{array}$ & $\begin{array}{l}\text { ICV (Poland) } \\
\text { PIF (India) } \\
\text { IBI (India) }\end{array}$ & $\begin{array}{l}=6.630 \\
=1.940 \\
=4.260\end{array}$ \\
\hline
\end{tabular}

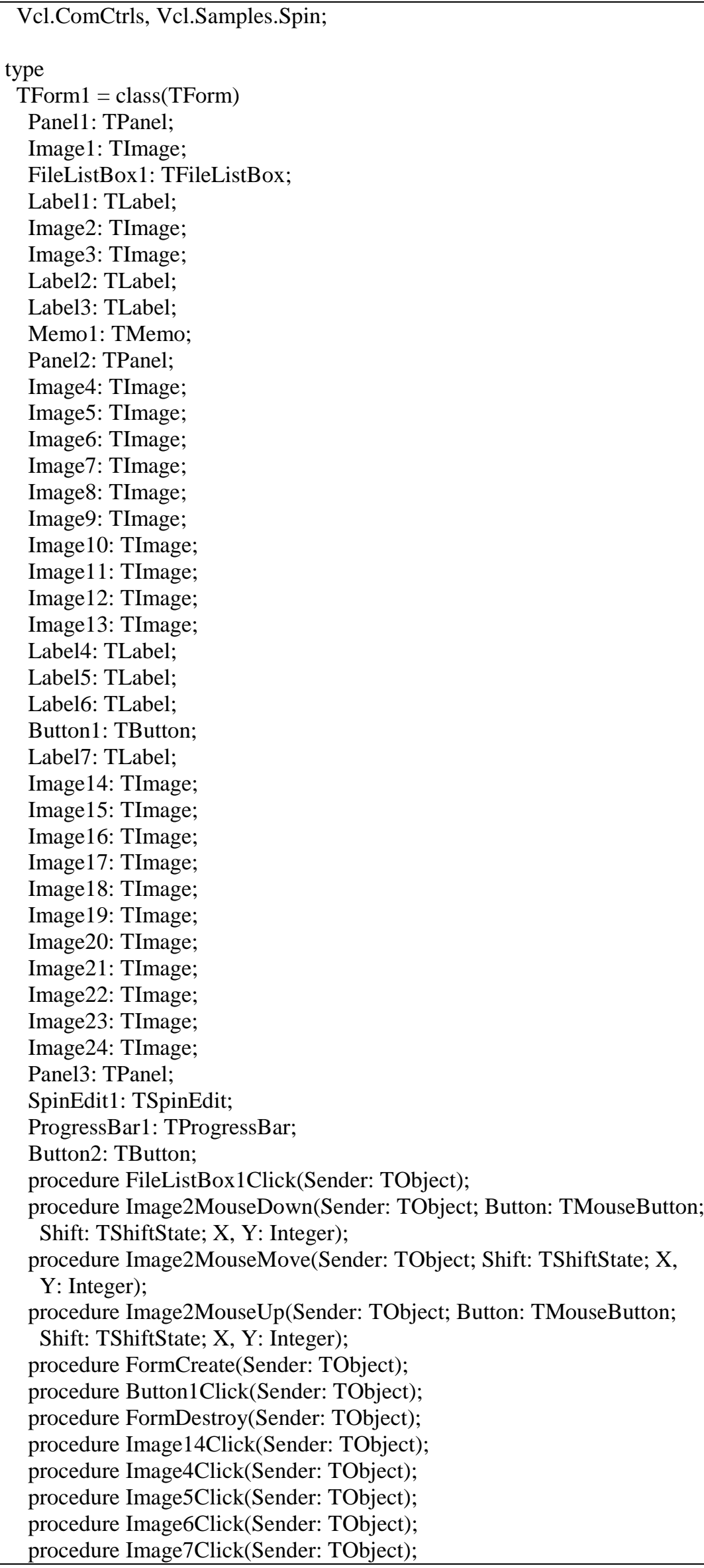

ISPC Theory and Practice, 


\begin{tabular}{|c|c|c|c|c|c|c|}
\hline Impact Factor: & $\begin{array}{l}\text { ISRA (India) } \\
\text { ISI (Dubai, UAF } \\
\text { GIF (Australia) } \\
\text { JIF }\end{array}$ & $\begin{array}{l}=1.344 \\
=0.829 \\
=0.564 \\
=1.500\end{array}$ & $\begin{array}{l}\text { SIS (USA) } \\
\text { PИНЦ (Russia) } \\
\text { ESJI (KZ) } \\
\text { SJIF (Morocco) }\end{array}$ & $\begin{array}{l}=0.912 \\
=0.234 \\
=1.042 \\
=2.031\end{array}$ & $\begin{array}{l}\text { ICV (Poland) } \\
\text { PIF (India) } \\
\text { IBI (India) }\end{array}$ & $\begin{array}{l}=6.630 \\
=1.940 \\
=4.260\end{array}$ \\
\hline
\end{tabular}

procedure Image8Click(Sender: TObject); procedure Image9Click(Sender: TObject); procedure Image10Click(Sender: TObject); procedure Image11Click(Sender: TObject); procedure Image12Click(Sender: TObject); procedure Image13Click(Sender: TObject); procedure Image15Click(Sender: TObject); procedure Image16Click(Sender: TObject); procedure Image17Click(Sender: TObject); procedure Image 18Click(Sender: TObject); procedure Image19Click(Sender: TObject); procedure Image20Click(Sender: TObject); procedure Image21Click(Sender: TObject); procedure Image22Click(Sender: TObject); procedure Image23Click(Sender: TObject); procedure Image24Click(Sender: TObject); procedure SpinEdit1Change(Sender: TObject); procedure Button2Click(Sender: TObject); private

\{Private declarations \} public

\{Public declarations \} end;

var

Form1: TForm1;

fname:string;

d:boolean;

i,x1,y1,maxi:integer; c:array[1..1000] of tcolor; b:array[0..1000] of tbitmap; implementation

$\{\$ \mathrm{R} * \mathrm{dfm}\}$

procedure TForm1.Button1Click(Sender: TObject);

var

$\mathrm{i}, \mathrm{j}, \mathrm{k}:$ integer; cc:tcolor;

pr:boolean;

begin

maxi: $=0$;

for $\mathrm{k}:=1$ to $1000 \mathrm{do}$

$\mathrm{b}[\mathrm{k}]$.Canvas.FillRect(rect $(0,0,620,620))$;

progressbar1.Max:=62;

for I := 0 to $620 \mathrm{do}$

begin

for $\mathrm{j}:=0$ to 620 do

begin

$\mathrm{cc}:=\mathrm{b}[0]$. Canvas.Pixels $[\mathrm{i}, \mathrm{j}]$;

pr:=false;

for $\mathrm{k}:=1$ to maxi do

if $\mathrm{c}[\mathrm{k}]=\mathrm{cc}$ then begin pr:=true; $\mathrm{b}[\mathrm{k}]$.Canvas.Pixels $[\mathrm{i}, \mathrm{j}]:=\mathrm{cc}$;

end;

if $\operatorname{not}(\mathrm{pr})$ then begin inc(maxi);c[maxi]:=cc;

end;

end; 


\begin{tabular}{|c|c|c|c|c|c|c|}
\hline Impact Factor: & $\begin{array}{l}\text { ISRA (India) } \\
\text { ISI (Dubai, UAF } \\
\text { GIF (Australia) } \\
\text { JIF }\end{array}$ & $\begin{array}{l}=1.344 \\
=0.829 \\
=0.564 \\
=1.500\end{array}$ & $\begin{array}{l}\text { SIS (USA) } \\
\text { PИНЦ (Russia) } \\
\text { ESJI (KZ) } \\
\text { SJIF (Morocco) }\end{array}$ & $\begin{array}{l}=0.912 \\
=0.234 \\
=1.042 \\
=2.031\end{array}$ & $\begin{array}{l}\text { ICV (Poland) } \\
\text { PIF (India) } \\
\text { IBI (India) }\end{array}$ & $\begin{array}{l}=6.630 \\
=1.940 \\
=4.260\end{array}$ \\
\hline
\end{tabular}

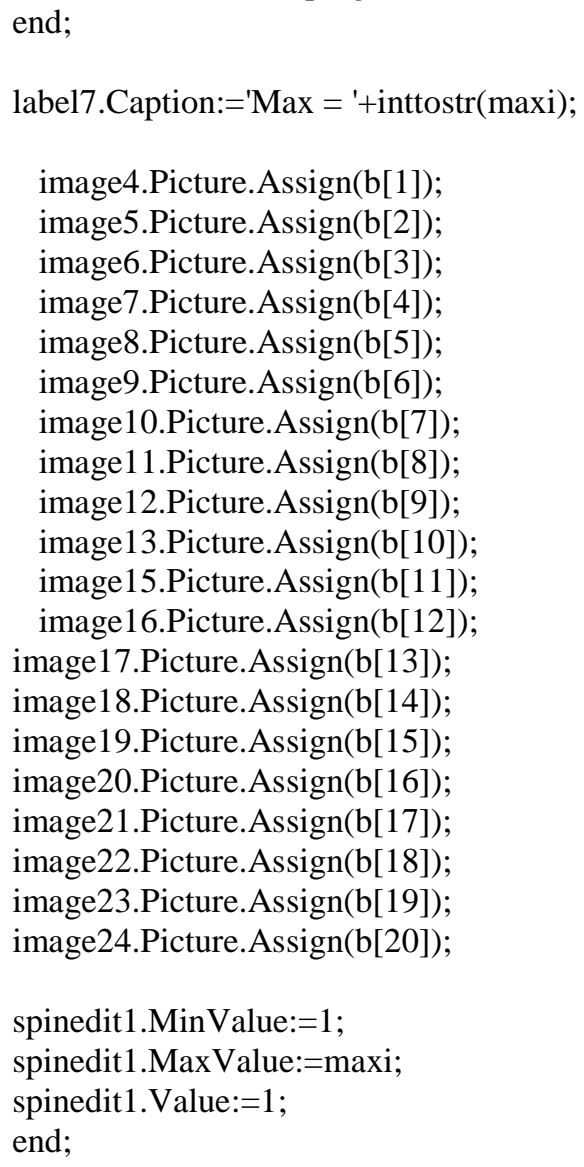




\begin{tabular}{l|lrl|l|ll} 
& ISRA (India) & $=\mathbf{1 . 3 4 4}$ & SIS (USA) & $=\mathbf{0 . 9 1 2}$ & ICV (Poland) & $=\mathbf{6 . 6 3 0}$ \\
Impact Factor: & ISI (Dubai, UAE) $=\mathbf{0 . 8 2 9}$ & PUHL (Russia) $=\mathbf{0 . 2 3 4}$ & PIF (India) & $=1.940$ \\
& GIF (Australia) & $\mathbf{0 . 5 6 4}$ & ESJI (KZ) & $=\mathbf{1 . 0 4 2}$ & IBI (India) & $=\mathbf{4 . 2 6 0}$ \\
& JIF & $\mathbf{1 . 5 0 0}$ & SJIF (Morocco) & $=\mathbf{2 . 0 3 1}$ & & \\
\hline
\end{tabular}

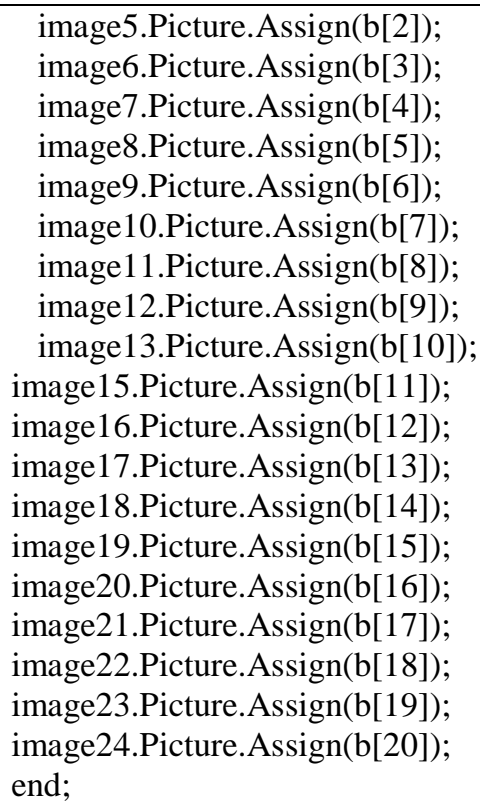




\begin{tabular}{|c|c|c|c|c|c|c|}
\hline Impact Factor: & $\begin{array}{l}\text { ISRA (India) } \\
\text { ISI (Dubai, UAE } \\
\text { GIF (Australia) } \\
\text { JIF }\end{array}$ & $\begin{array}{l}=1.344 \\
=0.829 \\
=0.564 \\
=1.500\end{array}$ & $\begin{array}{l}\text { SIS (USA) } \\
\text { PИНЦ (Russia) } \\
\text { ESJI (KZ) } \\
\text { SJIF (Morocco) }\end{array}$ & $\begin{array}{l}=0.912 \\
=0.234 \\
=1.042 \\
=2.031\end{array}$ & $\begin{array}{l}\text { ICV (Poland) } \\
\text { PIF (India) } \\
\text { IBI (India) }\end{array}$ & $\begin{array}{l}=6.630 \\
=1.940 \\
=4.260\end{array}$ \\
\hline
\end{tabular}

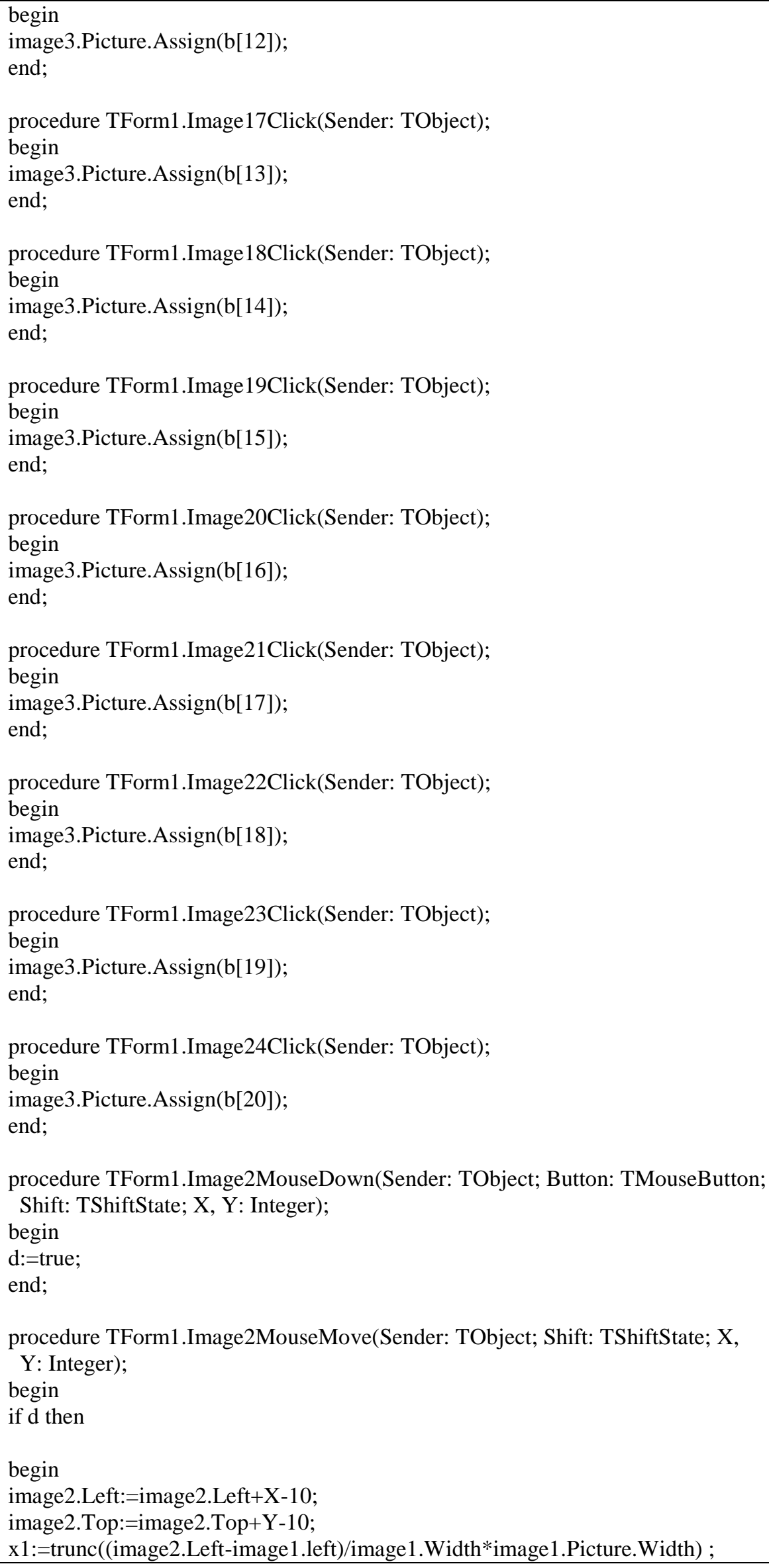

ISPC Theory and Practice, 


\begin{tabular}{|c|c|c|c|c|c|c|}
\hline Impact Factor: & $\begin{array}{l}\text { ISRA (India) } \\
\text { ISI (Dubai, UAE } \\
\text { GIF (Australia) } \\
\text { JIF }\end{array}$ & $\begin{array}{l}=1.344 \\
=0.829 \\
=0.564 \\
=1.500\end{array}$ & $\begin{array}{l}\text { SIS (USA) } \\
\text { PИНЦ (Russia) } \\
\text { ESJI (KZ) } \\
\text { SJIF (Morocco) }\end{array}$ & $\begin{array}{l}=0.912 \\
=0.234 \\
=1.042 \\
=2.031\end{array}$ & $\begin{array}{l}\text { ICV (Poland) } \\
\text { PIF (India) } \\
\text { IBI (India) }\end{array}$ & $\begin{array}{l}=6.630 \\
=1.940 \\
=4.260\end{array}$ \\
\hline
\end{tabular}

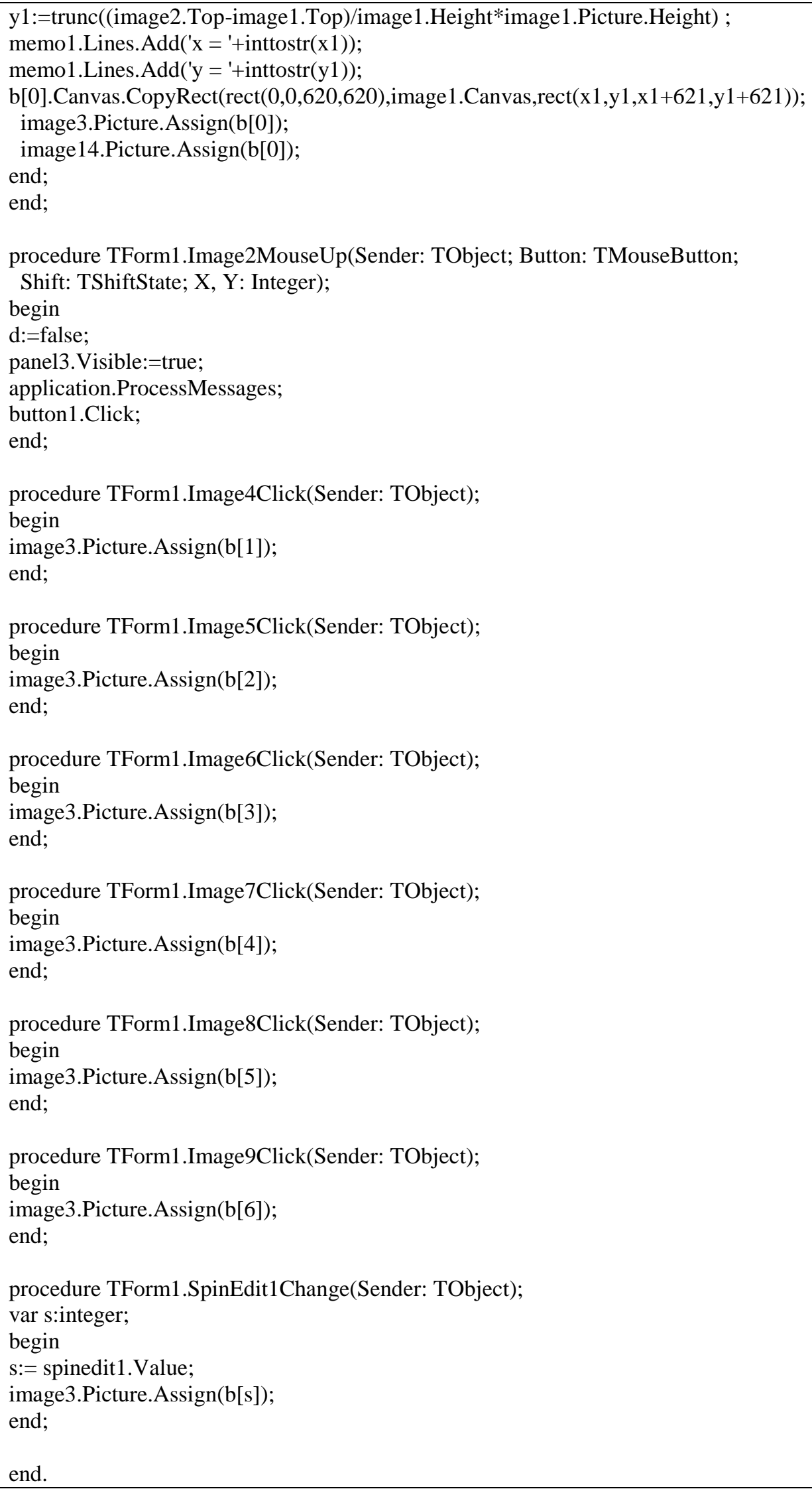

